\title{
A theory of superconductivity in cuprates
}

\author{
N.M.Plakida \\ Joint Institute for Nuclear Research, 141980 Dubna, Russia
}

Received July 11, 2005

\begin{abstract}
A microscopic theory of superconducting pairing mediated by antiferromagnetic (AFM) exchange and spin-fluctuations is developed within the effective $\boldsymbol{p}-\boldsymbol{d}$ Hubbard model for the $\mathrm{CuO}_{2}$ plane. It is proved that retardation effects for AFM exchange interaction are unimportant and result in pairing of all electrons in the conduction band and high $T_{\mathrm{c}}$ proportional to the Fermi energy. The spin-fluctuations caused by the kinematic interaction give an additional contribution to the $d$-wave pairing. $T_{\mathrm{c}}$ dependence on the hole concentration and lattice constants (or pressure) is studied. Small oxygen isotope shift of $\boldsymbol{T}_{\mathrm{c}}$ is explained. The data are compared with the results for the $t-J$ model.
\end{abstract}

Key words: high-temperature superconductivity, strong electron correlations, Hubbard model, antiferromagnetic exchange interaction, spin-fluctuations

PACS: 74.20.-z, 74.20.Mn, 74.72.-h

\section{Introduction}

A unique property of cuprates is their belonging to charge-transfer insulator with a small splitting energy between $3 d$ copper and $2 p$ oxygen levels and large Coulomb correlations in $3 d$ copper states. These result in a huge antiferromagnetic (AFM) superexchange interaction of the order of $J \simeq 1500 \mathrm{~K}$ which brings about a longrange AFM order in the undoped regime and causes strong AFM dynamical spin fluctuations in the superconducting state. The AFM spin fluctuations can also be responsible for anomalous normal state properties of cuprates (see, e.g. [1]) and for the superconducting pairing as proposed by Anderson [2]. In in a number of studies of the reduced one-band $t-J$ model (see, e.g. [3-6]) it was demonstrated that the instantaneous AFM exchange interaction mediates the $d$-wave pairing with a high $T_{\mathrm{c}}$. However, to prove the AFM pairing mechanism one has to consider the original two-band $p-d$ model for $\mathrm{CuO}_{2}$ layer [7] without reducing the interband hopping to the effective exchange interaction in one subband of the $t$ - $J$ model.

In this paper we describe a microscopic theory of superconductivity within the 
effective $p-d$ Hubbard model [8-10]. By applying the Mori-type projection technique to the matrix Green function in terms of the Hubbard operators, the Dyson equation is derived [11]. It is proved that in the mean-field approximation (MFA) the $d$-wave superconducting pairing mediated by the interband exchange interaction occurs similar to the $t-J$ model. The self-energy is calculated in the non-crossing approximation (or the self-consistent Born approximation) which gives an additional contribution to the $d$-wave pairing mediated by spin-fluctuations caused by the kinematic interaction in the intraband hopping. The results of numerical solution of the gap equation are presented for the superconducting $T_{\mathrm{c}}$ as a function of hole concentration and the superconducting gap as a function of the wave-vector [11]. Two remarkable features for cuprate superconductors which distinguish them from the conventional ones, i.e., the increase of $T_{\mathrm{c}}$ with pressure and small oxygen isotope shift of $T_{\mathrm{c}}$, are explained [6] as well. These results for the two-band $p-d$ model are compared with calculations for the $t-J$ model [5].

\section{Effective Hubbard model}

\subsection{Dyson equation}

We consider the original two-band $p-d$ model for the $\mathrm{CuO}_{2}$ layer [7] where two bonding oxygen orbitals $p_{x}$ and $p_{y}$ and the copper $3 d_{x^{2}-y^{2}}$ orbital are taken into account as shown in figure 1. By applying the sell-cluster perturbation theory [8-10]
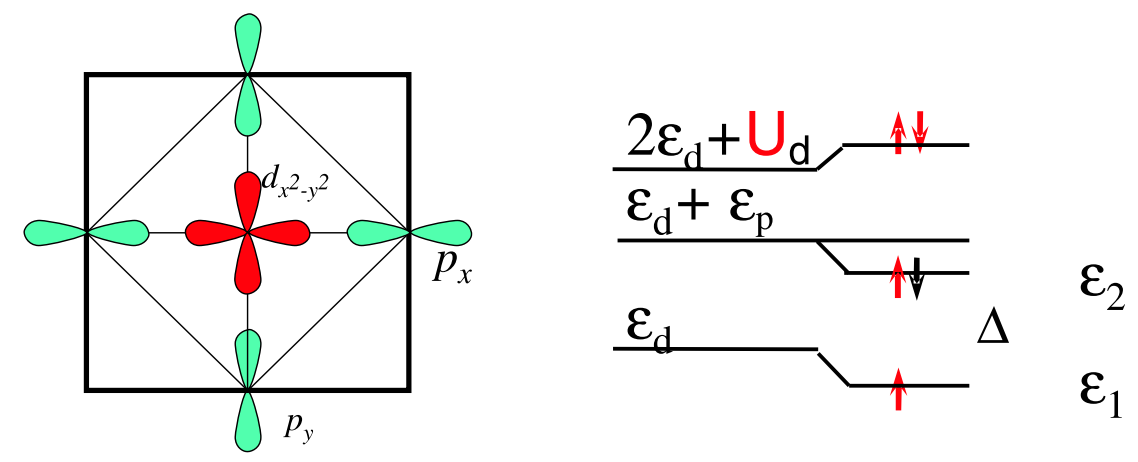

Figure 1. Effective two-band $p-d$ model for $\mathrm{CuO}_{2}$ layer [7].

we can reduce it to the effective two-band Hubbard model with the lower Hubbard subband occupied by one-hole $\mathrm{Cu} d$-like states and the upper Hubbard subband occupied by two-hole $p-d$ singlet states

$$
\begin{aligned}
H= & \varepsilon_{1} \sum_{i, \sigma} X_{i}^{\sigma \sigma}+\varepsilon_{2} \sum_{i} X_{i}^{22} \\
& +\sum_{i \neq j, \sigma}\left\{t_{i j}^{11} X_{i}^{\sigma 0} X_{j}^{0 \sigma}+t_{i j}^{22} X_{i}^{2 \sigma} X_{j}^{\sigma 2}+2 \sigma t_{i j}^{12}\left(X_{i}^{2 \bar{\sigma}} X_{j}^{0 \sigma}+\text { H.c. }\right)\right\},
\end{aligned}
$$

where $X_{i}^{n m}=|i n\rangle\langle i m|$ are the Hubbard operators for the four states $n, m=$ $|0\rangle,|\sigma\rangle,|2\rangle=|\uparrow \downarrow\rangle, \sigma= \pm 1 / 2=(\uparrow, \downarrow), \bar{\sigma}=-\sigma$. Here $\varepsilon_{1}=\varepsilon_{d}-\mu$ and $\varepsilon_{2}=2 \varepsilon_{1}+\Delta$ 
where $\mu$ is the chemical potential and $\Delta=\epsilon_{p}-\epsilon_{d}$ is the charge transfer energy (see [8]). The superscript 2 and 1 refers to the singlet and one-hole subbands, respectively. The hopping integrals are given by $t_{i j}^{\alpha \beta}=K_{\alpha \beta} 2 t \nu_{i j}$ where $t$ is the $p-d$ hybridization parameter and $\nu_{i j}$ are estimated as: $\nu_{1}=\nu_{j}{ }_{j \pm a_{x / y}} \simeq-0.14$, $\nu_{2}=\nu_{j}{ }_{j \pm a_{x} \pm a_{y}} \simeq-0.02$. The coefficients $K_{\alpha \beta}<1$, and for the singlet subband, e.g., we have $t_{\text {eff }} \simeq K_{22} 2 t \nu_{1} \simeq 0.14 t$ and the bandwidth $W=8 t_{\text {eff. }}$. If we take the standard parameters, $\Delta=2 t \simeq 3 \mathrm{eV}$ we get for the ratio $\Delta / W \simeq 2$ which shows that the Hubbard model (1) corresponds to the strong correlation limit. The chemical potential $\mu$ depends on the average electron occupation number

$$
n=\left\langle N_{i}\right\rangle=\sum_{\sigma}\left\langle X_{i}^{\sigma \sigma}\right\rangle+2\left\langle X_{i}^{22}\right\rangle
$$

where the number operator is $N_{i}=\sum_{\sigma} X_{i}^{\sigma \sigma}+2 X_{i}^{22}$. The Hubbard operators entering (1) obey the completeness relation

$$
X_{i}^{00}+X_{i}^{\uparrow \uparrow}+X_{i}^{\downarrow \downarrow}+X_{i}^{22}=1
$$

which rigorously preserves the constraint of no double occupancy of any quantum state $|i n\rangle$ at each lattice site $i$.

To discuss the superconducting pairing within the model Hamiltonian (1), we introduce the four-component Nambu operators $\widehat{X}_{i \sigma}$ and $\widehat{X}_{i \sigma}^{\dagger}$ and define the $4 \times 4$ matrix Green function (GF) [12]

$$
\tilde{G}_{i j \sigma}\left(t-t^{\prime}\right)=\left\langle\left\langle\widehat{X}_{i \sigma}(t) \mid \widehat{X}_{j \sigma}^{\dagger}\left(t^{\prime}\right)\right\rangle\right\rangle, \quad \tilde{G}_{i j \sigma}(\omega)=\left(\begin{array}{cc}
\widehat{G}_{i j \sigma}(\omega) & \widehat{F}_{i j \sigma}(\omega) \\
\widehat{F}_{i j \sigma}^{\dagger}(\omega) & -\widehat{G}_{j i \bar{\sigma}}(-\omega)
\end{array}\right),
$$

where $\widehat{X}_{i \sigma}^{\dagger}=\left(X_{i}^{2 \sigma} X_{i}^{\bar{\sigma} 0} X_{i}^{\bar{\sigma} 2} X_{i}^{0 \sigma}\right)$ and $\widehat{G}_{i j \sigma}$ and $\widehat{F}_{i j \sigma}$ are normal and anomalous $2 \times 2$ matrix components, respectively. By applying the projection technique for equation of motion method for GF (4), we derive the Dyson equation in $(\mathbf{q}, \omega)$ representation [11]:

$$
\left(\tilde{G}_{\sigma}(\mathbf{q}, \omega)\right)^{-1}=\left(\tilde{G}_{\sigma}^{0}(\mathbf{q}, \omega)\right)^{-1}-\tilde{\Sigma}_{\sigma}(\mathbf{q}, \omega), \quad \tilde{G}_{\sigma}^{0}(\mathbf{q}, \omega)=\left(\omega \tilde{\tau}_{0}-\tilde{E}_{\sigma}(\mathbf{q})\right)^{-1} \tilde{\chi}
$$

where $\tilde{\tau}_{0}$ is the $4 \times 4$ unity matrix and $\tilde{\chi}=\left\langle\left\{\widehat{X}_{i \sigma}, \widehat{X}_{i \sigma}^{\dagger}\right\}\right\rangle$. The zero-order GF within the generalized mean field approximation (MFA) is defined by the frequency matrix which in the site representation reads

$$
\tilde{E}_{i j \sigma}=\tilde{\mathcal{A}}_{i j \sigma} \tilde{\chi}^{-1}, \quad \tilde{\mathcal{A}}_{i j \sigma}=\left\langle\left\{\left[\widehat{X}_{i \sigma}, H\right], \widehat{X}_{j \sigma}^{\dagger}\right\}\right\rangle .
$$

The self-energy operator in the Dyson equation (5) in the projection technique method is defined by a proper part (having no single zero-order GF) of the manyparticle GF in the form

$$
\tilde{\Sigma}_{\sigma}(\mathbf{q}, \omega)=\tilde{\chi}^{-1}\left\langle\left\langle\widehat{Z}_{\sigma}^{(\mathrm{ir})} \mid \widehat{Z}_{\sigma}^{(\mathrm{ir}) \dagger}\right\rangle\right\rangle_{\mathbf{q}, \omega}^{(\mathrm{prop})} \tilde{\chi}^{-1} .
$$


Here the irreducible $\widehat{Z}$-operator is given by the equation: $\widehat{Z}_{\sigma}^{(\mathrm{ir})}=\left[\widehat{X}_{i \sigma}, H\right]-\sum_{l} \tilde{E}_{i l \sigma} \widehat{X}_{l \sigma}$ which follows from the orthogonality condition: $\left\langle\left\{\widehat{Z}_{\sigma}^{(\mathrm{ir})}, \widehat{X}_{j \sigma}^{\dagger}\right\}\right\rangle=0$. The equations (5)-(7) provide an exact representation for the GF (4). However, to calculate it one has to use approximations for the self-energy matrix (7) which describes the finite lifetime effects (i.e., the effects of inelastic scattering of electrons on spin and charge fluctuations).

\subsection{Mean-field approximation}

In the MFA the electronic spectrum and superconducting pairing are described by the zero-order GF in (5). By applying the commutation relations to the Hubbard operators we get for the frequency matrix (6):

$$
\tilde{\mathcal{A}}_{i j \sigma}=\left(\begin{array}{cr}
\widehat{\omega}_{i j \sigma} & \widehat{\Delta}_{i j \sigma} \\
\widehat{\Delta}_{j i \sigma}^{*} & -\widehat{\omega}_{j i \sigma}
\end{array}\right) .
$$

The normal component $\widehat{\omega}_{i j \sigma}$ defines quasiparticle spectra $\Omega_{1,2}(\mathbf{q})$ for two Hubbard subbands of the model in the normal state which have been studied in detail in [8]. As an example, in figure 2 and figure 3 the dispersion $\Omega_{1,2}(\mathbf{q})$ (solid lines) and the density of states (DOS) are shown for the undoped case, $n=1$, and for the overdoped case, $n=1.4$, respectively. For $n=1$ an insulating state is observed with the Fermi level (dotted line) being between the subbands with a dispersion defined by the next nearest neighbour hopping, while for $n=1.4$ the Fermi level is in the singlet subband with a dispersion defined by the nearest neighbour hopping.

$\operatorname{ENERGY}(\mathrm{eV})$

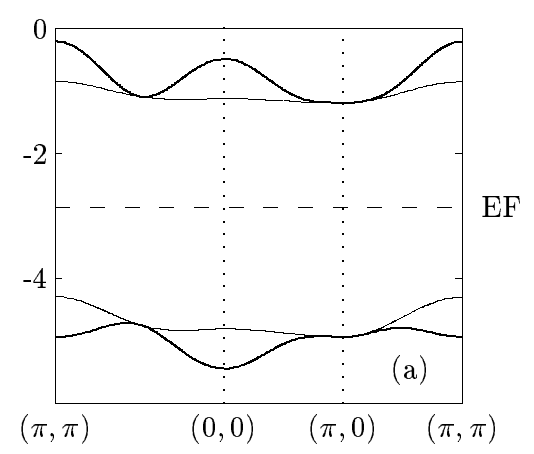

$\operatorname{DOS}($ states/eV)

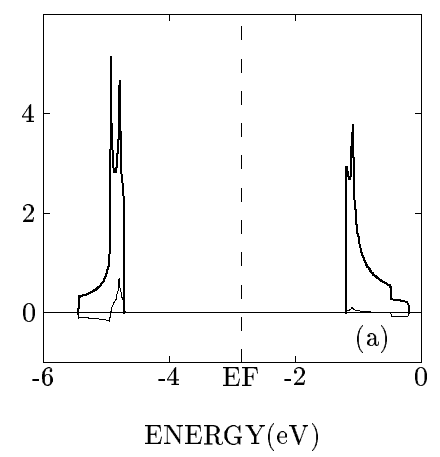

Figure 2. Hole dispersion curves along the symmetry directions (left panel) and the corresponding DOS (right panel) for the half-filled case, $n=1$, for the parameters $\Delta=2 t \simeq 3 \mathrm{eV}$ in the model (1) [8].

The anomalous component $\widehat{\Delta}_{i j \sigma}$ defines the gap functions for the singlet and one-hole subbands, respectively, $(i \neq j)$ :

$$
\Delta_{i j \sigma}^{22}=-2 \sigma t_{i j}^{12}\left\langle X_{i}^{02} N_{j}\right\rangle, \quad \Delta_{i j \sigma}^{11}=-2 \sigma t_{i j}^{12}\left\langle\left(2-N_{j}\right) X_{i}^{02}\right\rangle .
$$

Using the definitions of the Fermi annihilation operators: $c_{i \sigma}=X_{i}^{0 \sigma}+2 \sigma X_{i}^{\bar{\sigma} 2}$, we can write the anomalous average in (9) as $\left\langle c_{i \downarrow} c_{i \uparrow} N_{j}\right\rangle=\left\langle X_{i}^{0 \downarrow} X_{i}^{\downarrow 2} N_{j}\right\rangle=\left\langle X_{i}^{02} N_{j}\right\rangle$ 


\section{$\operatorname{ENERGY(eV)}$}

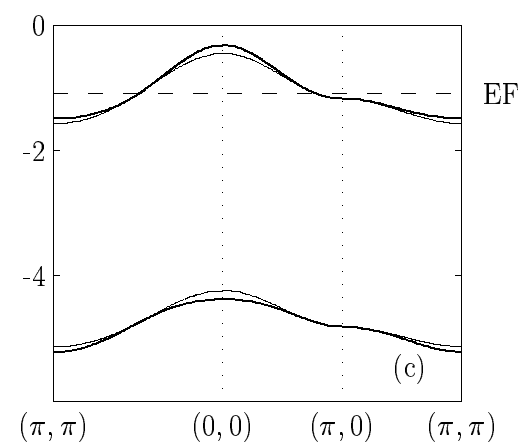

$\operatorname{DOS}($ states $/ \mathrm{eV})$

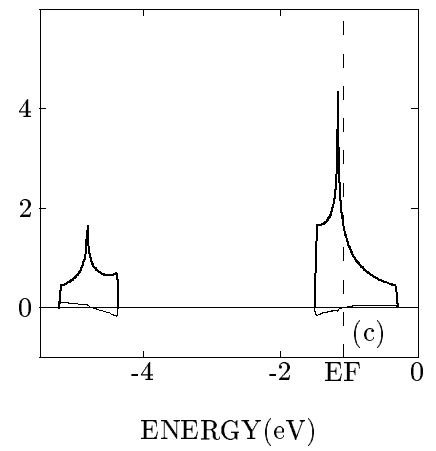

Figure 3. The same as in figure 2 for the overdoped case, $n=1.4[8]$.

since other products of the Hubbard operators vanish according to the multiplication rule for the Hubbard operators: $X_{i}^{\alpha \gamma} X_{i}^{\lambda \beta}=\delta_{\gamma, \lambda} X_{i}^{\alpha \beta}$. Therefore the anomalous correlation functions describe the pairing at one lattice site but in different Hubbard subbands.

The same anomalous correlation functions were obtained in MFA for the original Hubbard model in [13-15]. To calculate the anomalous correlation function $\left\langle c_{i \downarrow} c_{i \uparrow} N_{j}\right\rangle$ in $[13,15]$ the Roth procedure was applied based on a decoupling of the operators on the same lattice site in the time-dependent correlation function: $\left\langle c_{i \downarrow}(t) \mid c_{i \uparrow}\left(t^{\prime}\right) N_{j}\left(t^{\prime}\right)\right\rangle$. However, the decoupling of the Hubbard operators on the same lattice site is not unique (as has been really observed in $[13,15]$ ) and turns out to be unreliable. To escape uncontrollable decoupling, in [14] kinematical restrictions imposed on the correlation functions for the Hubbard operators were used which, however, have not produced a unique solution for superconducting equations either.

In our approach we perform a direct calculation of the correlation function $\left\langle X_{i}^{02} N_{j}\right\rangle$ without any decoupling by writing the equation of motion for the corresponding commutator $\mathrm{GF} L_{i j}\left(t-t^{\prime}\right)=\left\langle\left\langle X_{i}^{02}(t) \mid N_{j}\left(t^{\prime}\right)\right\rangle\right\rangle$ as follows:

$$
\left(\omega-\varepsilon_{2}\right) L_{i j}(\omega) \simeq 2 \delta_{i j}\left\langle X_{i}^{02}\right\rangle+\sum_{m \neq i, \sigma} 2 \sigma t_{i m}^{12}\left\{\left\langle\left\langle X_{i}^{0 \bar{\sigma}} X_{m}^{0 \sigma} \mid N_{j}\right\rangle\right\rangle_{\omega}-\left\langle\left\langle X_{i}^{\sigma 2} X_{m}^{\bar{\sigma} 2} \mid N_{j}\right\rangle\right\rangle_{\omega}\right\},
$$

where we neglected the intraband hopping $\left|t_{i m}^{\alpha \alpha}\right| \ll \varepsilon_{2} \simeq \Delta$. After applying the spectral theorem and neglecting exponentially small terms of the order of $\exp (-\Delta / T) \ll 1$, we obtain the following representation for the correlation function at sites $i \neq j$ for the singlet subband in the case of hole doping [11]:

$$
\left\langle X_{i}^{02} N_{j}\right\rangle=-\frac{1}{\Delta} \sum_{m \neq i, \sigma} 2 \sigma t_{i m}^{12}\left\langle X_{i}^{\sigma 2} X_{m}^{\bar{\sigma} 2} N_{j}\right\rangle \simeq-\frac{4 t_{i j}^{12}}{\Delta} 2 \sigma\left\langle X_{i}^{\sigma 2} X_{j}^{\bar{\sigma} 2}\right\rangle .
$$

The last equation is obtained in the two-site approximation, $m=j$, usually applied for the $t$ - $J$ model. The identity for the Hubbard operators, $X_{j}^{\bar{\sigma} 2} N_{j}=2 X_{j}^{\bar{\sigma} 2}$ was used as well. This finally permits us to write the gap function in (9) in the case of 
the hole doping as follows:

$$
\Delta_{i j \sigma}^{22}=-2 \sigma t_{i j}^{12}\left\langle X_{i}^{02} N_{j}\right\rangle=J_{i j}\left\langle X_{i}^{\sigma 2} X_{j}^{\bar{\sigma} 2}\right\rangle .
$$

This result is similar to the exchange interaction contribution to the pairing in the $t$ $J$ model with an exchange energy $J_{i j}=4\left(t_{i j}^{12}\right)^{2} / \Delta$. In the case of electron doping, an analogous calculation for the anomalous correlation function of the one-hole subband $\left\langle\left(2-N_{j}\right) X_{i}^{02}\right\rangle$ gives $\Delta_{i j \sigma}^{11}=J_{i j}\left\langle X_{i}^{0 \bar{\sigma}} X_{j}^{0 \sigma}\right\rangle$ for the gap function.

Therefore, we may conclude that the anomalous contributions to the zero-order GF (5) are just the conventional anomalous pairs of quasi-particles. Their pairing in MFA is mediated by the exchange interaction which has been studied in the $t$ $J$ model (see, e.g., [3,5]) and there are no new "composite operator excitations" ("cexons") proposed in [15].

\subsection{Self-energy}

The self-energy matrix (7) can be written in the form

$$
\tilde{\Sigma}_{i j \sigma}(\omega)=\tilde{\chi}^{-1}\left(\begin{array}{cc}
\widehat{M}_{i j \sigma}(\omega) & \widehat{\Phi}_{i j \sigma}(\omega) \\
\widehat{\Phi}_{i j \sigma}^{\dagger}(\omega) & -\widehat{M}_{i j \bar{\sigma}}(-\omega)
\end{array}\right) \tilde{\chi}^{-1}
$$

where the $2 \times 2$ matrices $\widehat{M}$ and $\widehat{\Phi}$ denote the normal and anomalous contributions to the self-energy, respectively.

The self-energy (13) is calculated below in the non-crossing (NCA) or the selfconsistent Born approximation (SCBA). In SCBA, the propagation of the Fermilike and Bose-like excitations in the many-particle GF in (13) are assumed to be independent of each other as shown schematically in figure 4. This approximation

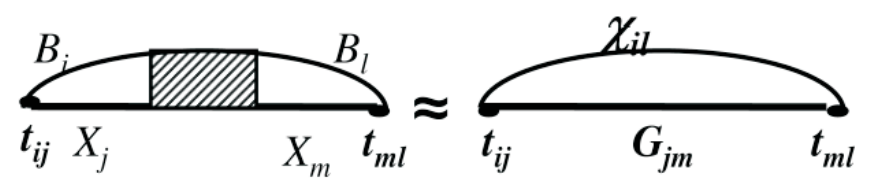

Figure 4. Self-consistent Born approximation for the self-energy (14).

is given by the decoupling of the corresponding operators in the time-dependent correlation functions for different lattice sites $(i \neq j, l \neq m)$ as follows

$$
\left\langle B_{i}(t) X_{j}(t) B_{l}\left(t^{\prime}\right) X_{m}\left(t^{\prime}\right)\right\rangle \simeq\left\langle X_{j}(t) X_{m}\left(t^{\prime}\right)\right\rangle\left\langle B_{i}(t) B_{l}\left(t^{\prime}\right)\right\rangle .
$$

Using the spectral representation for these correlation functions we get a closed system of equations for the GF (4) and the self-energy components (13) [11]. Below we explicitly write down only the anomalous part of the self-energy for the singlet band which is relevant in the further discussion:

$$
\begin{aligned}
\Phi_{\sigma}^{22}(\mathbf{q}, \omega) & =\frac{1}{N} \sum_{\mathbf{k}}|t(\mathbf{k})|^{2} \int_{-\infty}^{+\infty} \int_{-\infty}^{+\infty} \frac{\mathrm{d} \omega_{1} \mathrm{~d} \omega_{2}}{\omega-\omega_{1}-\omega_{2}} \frac{1}{2}\left(\tanh \frac{\omega_{1}}{2 T}+\operatorname{coth} \frac{\omega_{2}}{2 T}\right) \\
& \times \chi_{\mathrm{s}}^{\prime \prime}\left(\mathbf{q}-\mathbf{k}, \omega_{2}\right)\left\{-(1 / \pi) \operatorname{Im}\left[K_{22}^{2} F_{\sigma}^{22}\left(\mathbf{k}, \omega_{1}\right)-K_{21}^{2} F_{\sigma}^{11}\left(\mathbf{k}, \omega_{1}\right)\right]\right\} .
\end{aligned}
$$


The kinematic interaction for the nearest and the second neighbors is given by $t(\mathbf{k})=t_{1}(\mathbf{k})+t_{2}(\mathbf{k})=8 t\left[\nu_{1} \gamma(\mathbf{k})+\nu_{2} \gamma^{\prime}(\mathbf{k})\right]$, where $\gamma(\mathbf{k})=(1 / 2)\left(\cos k_{x}+\cos k_{y}\right)$ and $\gamma^{\prime}(\mathbf{k})=\cos k_{x} \cos k_{y}$. The pairing interaction is mediated by spin-fluctuations defined by the susceptibility $\chi_{\mathbf{s}}^{\prime \prime}(\mathbf{q}, \omega)=-(1 / \pi) \operatorname{Im}\left\langle\left\langle\mathbf{S}_{q} \mid \mathbf{S}_{-q}\right\rangle\right\rangle_{\omega+\mathrm{i} \delta}$ which comes from the bosonic correlation functions $\left\langle B_{i}(t) B_{l}\left(t^{\prime}\right)\right\rangle$ in (14).

For the hole doped case, at frequencies $\left|\omega, \omega_{1}\right| \ll \omega_{s} \ll W$ close to the Fermi surface (FS) $\left(\omega_{s} \leqslant J\right.$ is a characteristic spin-fluctuation energy) we can use the weak coupling approximation (WCA) to calculate the first term in the self-energy (15). The contribution from the second term $F_{\sigma}^{11}\left(\mathbf{k}, \omega_{1}\right)$ is rather small since the one-hole band lies below the FS at the energy of the order of $\Delta \gg W$. Neglecting it and taking into account the contribution from the exchange interaction in MFA (12), we arrive at the following equation for the superconducting gap in the singlet subband:

$$
\Phi^{22}(\mathbf{q})=\frac{1}{N} \sum_{\mathbf{k}}\left[J(\mathbf{k}-\mathbf{q})-K_{22}^{2} \lambda(\mathbf{k}, \mathbf{q}-\mathbf{k})\right] \frac{\Phi^{22}(\mathbf{k})}{2 E_{2}(\mathbf{k})} \tanh \frac{E_{2}(\mathbf{k})}{2 T},
$$

where $\lambda(\mathbf{k}, \mathbf{q}-\mathbf{k})=|t(\mathbf{k})|^{2} \chi_{s}(\mathbf{q}-\mathbf{k}, \omega=0)>0$. The quasiparticle energy in the singlet band is given by $E_{2}(\mathbf{k})=\left[\Omega_{2}(\mathbf{k})^{2}+\Phi^{22}(\mathbf{k})^{2}\right]$, where $\Omega_{2}(\mathbf{k})$ is the quasiparticle energy in the normal state as shown in figure 3. Similar considerations hold true for an electron doped system, $n \leqslant 1$ when the chemical potential lies in the onehole band, $\mu \simeq 0$. In that case, the WCA equation for the gap $\Phi^{11}(\mathbf{q})$ is quite similar to (16).

\section{Numerical results and discussion}

To solve the gap equation (16) we used the following model for the static spinfluctuation susceptibility:

$$
\chi_{s}(\mathbf{q}, 0)=\frac{\chi_{0}}{1+\xi^{2}[1+\gamma(\mathbf{q})]},
$$

where $\xi$ is the AFM correlation length and the constant $\chi_{0}=3(2-n) /\left(2 \pi \omega_{s} C_{1}\right)$ with $C_{1}=(1 / N) \sum_{\mathbf{q}}\left\{1+\xi^{2}[1+\gamma(\mathbf{q})]\right\}^{-1}$ is defined from the normalization condition: $(1 / N) \sum_{i}\left\langle\mathbf{S}_{\mathbf{i}} \mathbf{S}_{\mathbf{i}}\right\rangle=(3 / 4)(1-|1-n|)$. Let us first estimate the superconducting transition temperature $T_{\mathrm{c}}$ by solving the gap equation (16) for a model $d$-wave gap function $\Phi^{22}(\mathbf{q})=\varphi_{d}\left(\cos q_{x}-\cos q_{y}\right) \equiv \varphi_{d} \eta(\mathbf{q})$ in the standard logarithmic approximation in the limit of weak coupling. Integrating both sides of (16) over $\mathbf{q}$ multiplied by $\eta(\mathbf{q})$ results in the following equation for $T_{\mathrm{c}}$ :

$$
1=\frac{1}{N} \sum_{\mathbf{k}}\left[J \eta(\mathbf{k})^{2}+\lambda_{s}(4 \gamma(\mathbf{k}))^{2} \eta(\mathbf{k})^{2}\right] \frac{1}{2 \Omega_{2}(\mathbf{k})} \tanh \frac{\Omega_{2}(\mathbf{k})}{2 T_{\mathrm{c}}},
$$

where $\lambda_{s} \simeq t_{\text {eff }}^{2} / \omega_{s}$. For the exchange interaction mediated by the interband hopping with large energy transfer $\Delta \gg W$ the retardation effects are negligible which results in the coupling of all electrons in a broad energy shell of the order of the bandwidth $W$ and high $T_{\mathrm{c}}[6]$ :

$$
T_{\mathrm{c}} \simeq \sqrt{\mu(W-\mu)} \exp \left(-1 / \lambda_{\mathrm{ex}}\right)
$$


where $\lambda_{\mathrm{ex}} \simeq J N(\delta)$ is an effective coupling constant for the exchange interaction $J$ and the average density $N(\delta)$ of electronic states for doping $\delta$. By taking into account both contributions we can write the following estimation for $T_{\mathrm{c}}$ :

$$
T_{\mathrm{c}} \simeq \omega_{s} \exp \left(-\frac{1}{\tilde{\lambda}_{\mathrm{sf}}}\right), \quad \tilde{\lambda}_{\mathrm{sf}}=\lambda_{\mathrm{sf}}+\frac{\lambda_{\mathrm{ex}}}{1-\lambda_{\mathrm{ex}} \ln \left(\mu / \omega_{s}\right)}
$$

where $\lambda_{\mathrm{sf}} \simeq \lambda_{\mathrm{s}} N\left(E_{\mathrm{F}}\right)$ is the coupling constant for the spin-fluctuation pairing. By taking $\mu=W / 2 \simeq 0.35 \mathrm{eV}, \omega_{s} \simeq J \simeq 0.13 \mathrm{eV}$ and $\lambda_{\mathrm{sf}} \simeq \lambda_{\mathrm{ex}}=0.2$ for estimation we get $\tilde{\lambda}_{\mathrm{sf}} \simeq 0.2+0.25=0.45$ and $T_{\mathrm{c}} \simeq 160 \mathrm{~K}$, while only the spin-fluctuation pairing gives $T_{\mathrm{c}}^{0} \simeq \omega_{s} \exp \left(-1 / \lambda_{\mathrm{sf}}\right) \simeq 10 \mathrm{~K}$.

The results of numerical solution of the gap equation (16) are shown in figure 5 for the superconducting transition temperature $T_{c}(\delta)[11]$. The following parameters are used: $\xi=3, J=0.4 t_{\mathrm{eff}}, \omega_{s}=0.15 \mathrm{eV}$ and $t_{\mathrm{eff}}=K_{22} 2 t \nu_{1} \simeq 0.2 \mathrm{eV}$. The maximum
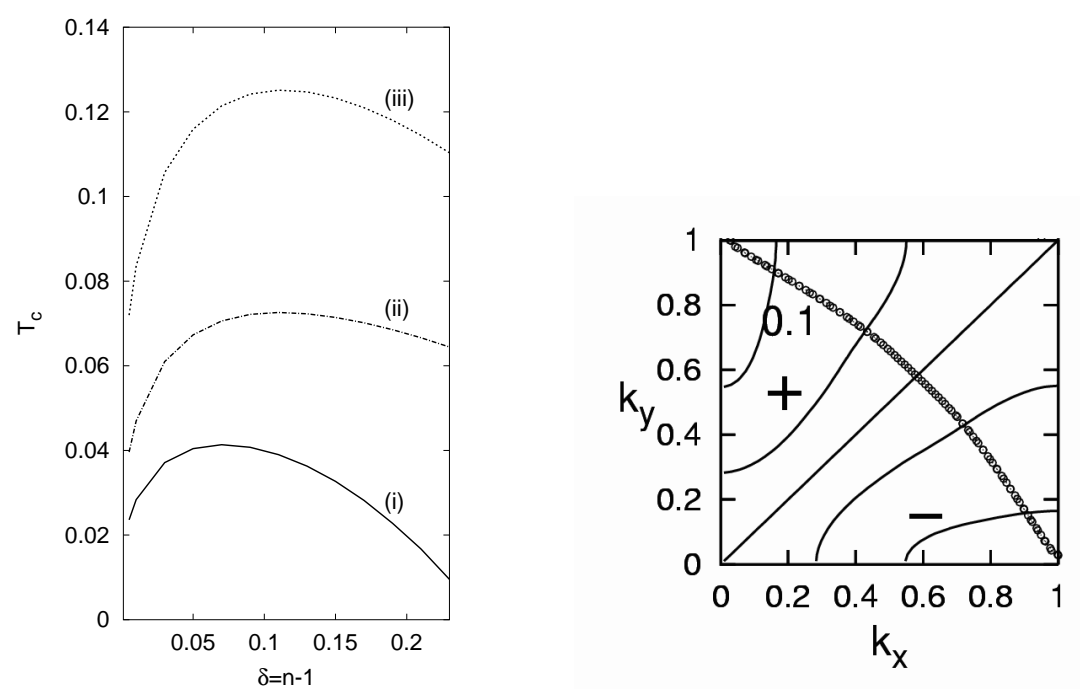

Figure 5. Left panel: superconducting $T_{c}(\delta)$ (in units of $t_{\text {eff }} \simeq 0.2 \mathrm{eV}$ ) for (i) spinfluctuation interaction (solid line), (ii) exchange interaction (dashed line), (iii) for the both contributions (dotted line). Right panel: wave-vector dependence of the gap function $\Phi^{22}(\mathbf{k})$ over the first quadrant of the BZ at optimum doping $(\delta=0.13)$. The circles plot the Fermi surface. The $+/-$ denote gap signs inside the octants [11].

$T_{\mathrm{c}} \sim 280 \mathrm{~K}$ (dotted line) is achieved for the chemical potential $\mu=E_{\mathrm{F}} \simeq W / 2$ at the optimal doping $\delta_{\mathrm{opt}} \simeq 0.12$. The spin-fluctuation interaction produces much lower $T_{\mathrm{c}}$ (solid line) since it couples the holes in a narrow energy shell, $\omega_{s} \ll E_{\mathrm{F}}$, near the Fermi surface (FS). This interaction is rather weak at the FS close to the AF zone boundary along the lines $\left|k_{x}\right|+\left|k_{y}\right|=\pi$ where the main contribution coming from the nearest neighbor hopping vanishes: $t_{1}(\mathbf{k}) \propto \gamma\left(k_{x},\left|k_{y}\right|=\pi-\left|k_{x}\right|\right)=0$.

We can confirm the AFM pairing mechanism by considering the $T_{\mathrm{c}}$ dependence on pressure or lattice constants. While in electron-phonon superconductors, $T_{\mathrm{c}}$ decreases under pressure, in cuprates, $T_{\mathrm{c}}$ increases with compression of the in-plane lattice constant $a$. In particular, in mercury superconductors $\mathrm{d} T_{\mathrm{c}} / \mathrm{d} a \simeq-1.35 \times 10^{3} \mathrm{~K} / \AA[16]$ 
and for $\mathrm{Hg}-1201$ compound we get $\mathrm{d} \ln T_{\mathrm{c}} / \mathrm{d} \ln a \simeq-50$. From (19) we get an estimate:

$$
\frac{\mathrm{d} \ln T_{\mathrm{c}}}{\mathrm{d} \ln a} \simeq \frac{\mathrm{d} \ln T_{\mathrm{c}}}{\mathrm{d} \ln J} \frac{\mathrm{d} \ln J}{\mathrm{~d} \ln a} \simeq-\frac{14}{\lambda} \simeq-47
$$

which is quite close to the experimentally observed one. Here we use $\lambda=J N(\delta) \simeq 0.3$ and take into account that for the exchange interaction we can use an estimate $J(a) \propto t_{p d}^{4}$ where $t_{p d} \propto 1 /(a)^{7 / 2}$ for the $p-d$ hybridization [17].

Concerning an oxygen isotope effect in cuprates, on substituting the ${ }^{18} \mathrm{O}$ oxygen for ${ }^{16} \mathrm{O}$, we can also estimate it from (19). By using the experimentally observed isotope shift for the Néel temperature in $\mathrm{La}_{2} \mathrm{CuO}_{4}[18]: \alpha_{\mathrm{N}}=-\left(\mathrm{d} \ln T_{\mathrm{N}} / \mathrm{d} \ln M\right) \simeq$ $(\mathrm{d} \ln J / \mathrm{d} \ln M) \simeq 0.05$ we obtain

$$
\alpha_{\mathrm{c}}=-\frac{\mathrm{d} \ln T_{\mathrm{c}}}{\mathrm{d} \ln M}=-\frac{\mathrm{d} \ln T_{\mathrm{c}}}{\mathrm{d} \ln J} \frac{\mathrm{d} \ln T_{\mathrm{N}}}{\mathrm{d} \ln M} \simeq \frac{\alpha_{\mathrm{N}}}{\lambda} \simeq 0.16
$$

for $\lambda \simeq 0.3$ which is close to experiments: $\alpha_{\mathrm{c}}=-\mathrm{d} \ln T_{\mathrm{c}} / \mathrm{d} \ln M \leqslant 0.1$.

\section{Comparison with the $t-J$ model}

Now we compare the results for the original two-band $p-d$ model for $\mathrm{CuO}_{2}$ layer (1) with the calculations for the $t-J$ in [5]. In that paper, a full self-consistent numerical solution for the normal and anomalous GF in the Dyson equation was performed in the strong-coupling limit allowing for the quasiparticle renormaization and finite life-time effects caused by the self-energy operators which were neglected in the above calculations for the Hubbard model.

In the limit of strong correlations the interband hopping in the model (1) can be excluded by perturbation theory which results in the effective $t$ - $J$ model

$$
H_{t-J}=-\sum_{i \neq j, \sigma} t_{i j} X_{i}^{\sigma 0} X_{j}^{0 \sigma}-\mu \sum_{i \sigma} X_{i}^{\sigma \sigma}+\frac{1}{4} \sum_{i \neq j, \sigma} J_{i j}\left(X_{i}^{\sigma \bar{\sigma}} X_{j}^{\bar{\sigma} \sigma}-X_{i}^{\sigma \sigma} X_{j}^{\bar{\sigma} \bar{\sigma}}\right)
$$

where only the lower Hubbard subband is considered with the hopping energy $t_{i j}=$ $-t_{i j}^{11}$. The exclusion of the interband hopping results in the instantaneous exchange interaction $J_{i j}=4\left(t_{i j}^{12}\right)^{2} / \Delta$. The superconducting pairing within the model (23) can be studied by considering the matrix GF for the lower Hubbard subband in terms of the Nambu operators: $\Psi_{i \sigma}$ and $\Psi_{i \sigma}^{+}=\left(X_{i}^{\sigma 0} X_{i}^{0 \bar{\sigma}}\right)$ :

$$
\widehat{G}_{i j, \sigma}\left(t-t^{\prime}\right)=\left\langle\left\langle\Psi_{i \sigma}(t) \mid \Psi_{j \sigma}^{+}\left(t^{\prime}\right)\right\rangle\right\rangle, \quad \widehat{G}_{i j \sigma}(\omega)=Q\left(\begin{array}{ll}
G_{i j \sigma}^{11}(\omega) & G_{i j \sigma}^{12}(\omega) \\
G_{i j \sigma}^{21}(\omega) & G_{i j \sigma}^{22}(\omega)
\end{array}\right) .
$$

Here we introduced the Hubbard factor $Q=1-n / 2$ depending on the average number of electrons $n=\sum_{\sigma}\left\langle X_{i}^{\sigma \sigma}\right\rangle$.

By applying the projection technique as described above we get the Dyson equation which can be written in the Eliashberg notation as

$$
\widehat{G}_{\sigma}(\mathbf{k}, \omega)=Q \frac{\omega Z_{\sigma}(\mathbf{k}, \omega) \widehat{\tau}_{0}+\left(E_{\sigma}(\mathbf{k})+\xi_{\sigma}(\mathbf{k}, \omega)-\tilde{\mu}\right) \widehat{\tau}_{3}+\Phi_{\sigma}(\mathbf{k}, \omega) \widehat{\tau_{1}}}{\left(\omega Z_{\sigma}(\mathbf{k}, \omega)\right)^{2}-\left(E_{\sigma}(\mathbf{k})+\xi_{\sigma}(\mathbf{k}, \omega)-\tilde{\mu}\right)^{2}-\left|\Phi_{\sigma}(\mathbf{k}, \omega)\right|^{2}},
$$


where $\widehat{\tau}_{i}$ are the Pauli matrices. The quasiparticle energy $E_{\sigma}(\mathbf{k})$ in the normal state and the renormalized chemical potential $\tilde{\mu}=\mu-\delta \mu$ are calculated in the MFA as discussed above (for details see [5]). The frequency-dependent functions

$\omega\left(1-Z_{\sigma}(\mathbf{k}, \omega)\right)=\frac{1}{2}\left[\Sigma_{\sigma}^{11}(\mathbf{k}, \omega)+\Sigma_{\sigma}^{22}(\mathbf{k}, \omega)\right], \quad \xi_{\sigma}(\mathbf{k}, \omega)=\frac{1}{2}\left[\Sigma_{\sigma}^{11}(\mathbf{k}, \omega)-\Sigma_{\sigma}^{22}(\mathbf{k}, \omega)\right]$

are defined by the normal components of the self-energy $\Sigma_{\sigma}^{22}(\mathbf{k}, \omega)=-\Sigma_{\bar{\sigma}}^{11}(\mathbf{k},-\omega)$. The gap function is specified by the equation:

$$
\Phi_{\sigma}(\mathbf{k}, \omega)=\Delta_{\sigma}(\mathbf{k})+\Sigma_{\sigma}^{12}(\mathbf{k}, \omega), \quad \Delta_{\sigma}(\mathbf{k})=\frac{1}{N Q} \sum_{\mathbf{q}} J(\mathbf{k}-\mathbf{q})\left\langle X_{-\mathbf{q}}^{0 \bar{\sigma}} X_{\mathbf{q}}^{0 \sigma}\right\rangle
$$

The self-energy is calculated in SCBA (14) as in the Hubbard model:

$$
\begin{aligned}
\Sigma_{\sigma}^{11(12)}(\mathbf{k}, \omega)= & \frac{1}{N} \sum_{q} g^{2}(\mathbf{q}, \mathbf{k}-\mathbf{q}) \int_{-\infty}^{+\infty} \int_{-\infty}^{+\infty} \frac{\mathrm{d} z \mathrm{~d} \Omega}{\omega-z-\Omega} \frac{1}{2}\left(\tanh \frac{z}{2 T}+\operatorname{coth} \frac{\Omega}{2 T}\right) \\
& \times A_{\sigma}^{11(12)}(\mathbf{q}, z)\left[-(1 / \pi) \operatorname{Im} D^{ \pm}(\mathbf{k}-\mathbf{q}, \Omega+\mathrm{i} \delta)\right]
\end{aligned}
$$

where the interaction $g(\mathbf{q}, \mathbf{k}-\mathbf{q})=t(\mathbf{q})-1 / 2 \cdot J(\mathbf{k}-\mathbf{q})$ and the spectral densities are defined by the corresponding $\mathrm{GF}$ :

$$
A_{\sigma}^{11(12)}(\mathbf{q}, z)=-\frac{1}{\pi} \operatorname{Im} G_{\sigma}^{11(12)}(\mathbf{q}, z+\mathrm{i} \delta) .
$$

The electron-electron interaction is caused by the spin-charge fluctuations defined by the boson-like commutator GF: $D^{ \pm}(\mathbf{q}, \Omega)=\langle\langle\mathbf{S}(\mathbf{q}) \mid \mathbf{S}(-\mathbf{q})\rangle\rangle_{\Omega} \pm 1 / 4\langle\langle n(\mathbf{q})|$ $n(-\mathbf{q})\rangle\rangle_{\Omega}$ as in the Hubbard model.

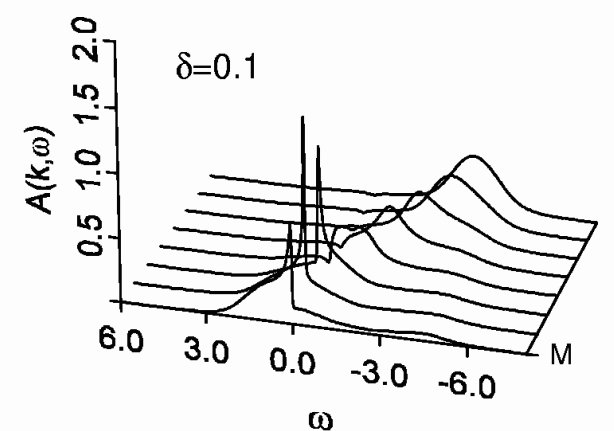

Figure 6. Spectral density $A_{\sigma}^{11}(\mathbf{q}, \omega)(28)$ (left panel) and $\operatorname{Im} \Sigma_{\sigma}^{11}(\mathbf{k}, \omega+\mathrm{i} \delta)(27)$ (right panel) along the symmetry direction $\mathrm{M}(\pi, \pi) \rightarrow \Gamma(00)$ at hole concentration $\delta=0.1$. Energy $\omega$ is measured in units of $t$, with $J=0.4 t$ [5].

As we see, the equation for the self-energy (27) is similar to (15) obtained for the Hubbard model if we disregard in the latter the small contribution from the second 
subband $\propto F_{\sigma}^{11}\left(\mathbf{k}, \omega_{1}\right)$. However, contrary to the gap equation (16) in the WCA for the Hubbard model, for the $t-J$ model in the equation (26) the frequency-dependent self-energy contribution $\Sigma_{\sigma}^{12}(\mathbf{k}, \omega)(27)$ is taken into account. Moreover, in [5] for the $t-J$ model a full self-consistent solution for the normal GF $G_{\sigma}^{11}(\mathbf{k}, \omega)$ in $(25)$ and for the corresponding self-energy $\Sigma_{\sigma}^{11}(\mathbf{k}, \omega)$ (27) was performed. The results of the calculations are shown in figure 6 in the left panel for the spectral density $A_{\sigma}^{11}(\mathbf{q}, \omega)(28)$ and in the right panel for the $\operatorname{Im} \Sigma_{\sigma}^{11}(\mathbf{k}, \omega+\mathrm{i} \delta)$ along the symmetry direction $\mathrm{M}(\pi, \pi) \rightarrow \Gamma(00)$ in the BZ. These results for the hole concentration $\delta=0.1$ and the AFM correlation length $\xi=3$ in the model spin susceptibility (17) demonstrate quasiparticle-like peaks only in the vicinity of the Fermi level and anomalous behavior for the self-energy $\operatorname{Im} \Sigma_{\sigma}^{11}(\mathbf{k}, \omega+\mathrm{i} \delta) \propto \omega$ close to the Fermi level. The occupation number $N(\mathbf{k})=(1 / Q)\left\langle X_{\mathbf{k}}^{\sigma \sigma}\right\rangle$ shown in the left panel of figure 7 , reveals only a small drop at the Fermi level which is generic for strongly correlated systems (the calculations are done at finite temperature and the character of the drop cannot be disclosed).
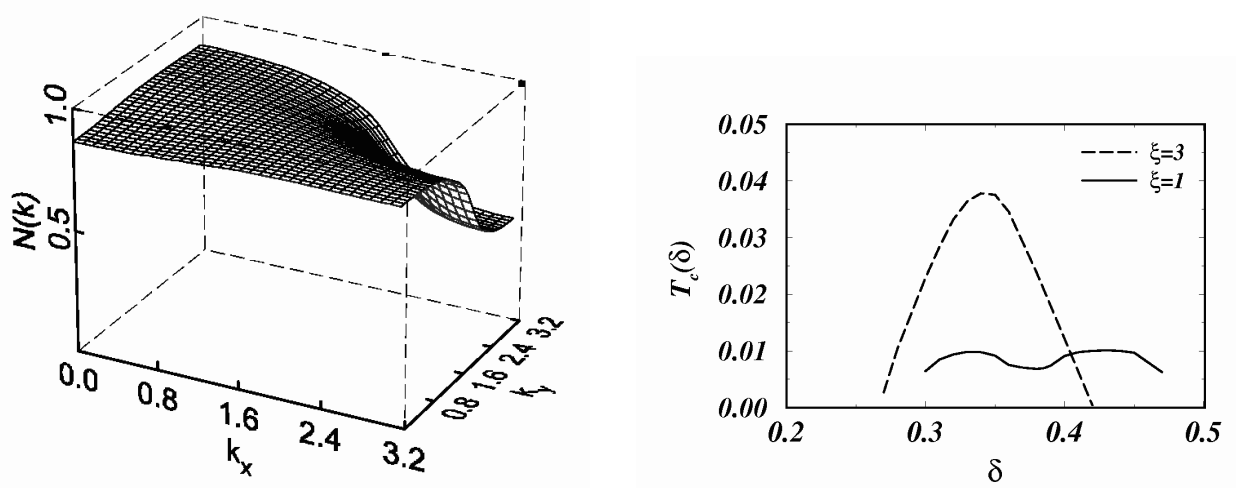

Figure 7. Left panel: occupation numbers $N(\mathbf{k})=(1 / Q)\left\langle X_{\mathbf{k}}^{\sigma 0} X_{\mathbf{k}}^{0 \sigma}\right\rangle$. Right panel: $T_{\mathrm{c}}(\delta)$ for the AFM correlation length $\xi=1$ (full line) and $\xi=3$ (dashed line) [5].

The superconducting $T_{\mathrm{c}}$ was calculated from a linearized gap equation which was solved by direct diagonalization in $(\mathbf{k}, \omega)$-space:

$$
\begin{aligned}
\Phi_{\sigma}\left(\mathbf{k}, i \omega_{n}\right)= & \frac{T}{N} \sum_{\mathbf{q}} \sum_{m}\left\{J(\mathbf{k}-\mathbf{q})+\lambda_{12}\left(\mathbf{q}, \mathbf{k}-\mathbf{q} \mid \mathrm{i} \omega_{n}-\mathrm{i} \omega_{m}\right)\right\} \\
& \times G_{\sigma}^{11}\left(\mathbf{q}, \mathrm{i} \omega_{m}\right) G_{\bar{\sigma}}^{11}\left(\mathbf{q},-\mathrm{i} \omega_{m}\right) \Phi_{\sigma}\left(\mathbf{q}, \mathrm{i} \omega_{m}\right)
\end{aligned}
$$

where the interaction function $\lambda_{12}\left(\mathbf{q}, \mathbf{k}-\mathbf{q} \mid \mathrm{i} \omega_{\nu}\right)=g^{2}(\mathbf{q}, \mathbf{k}-\mathbf{q}) D^{-}\left(\mathbf{k}-\mathbf{q}, \mathrm{i} \omega_{\nu}\right)$ and the Matsubara frequencies $i \omega_{n}=\mathrm{i} \pi T(2 n+1)$ were introduced. The doping dependence of superconducting $T_{\mathrm{c}}(\delta)$ is shown in the right panel of figure 7 for the AFM correlation length $\xi=1$ (full line) and $\xi=3$ (dashed line). Also the eigenfunctions $\Phi_{\sigma}\left(\mathbf{k}, \mathrm{i} \omega_{n}\right)$ of the equation (29) were determined which unambiguously demonstrated the $d$-wave character of superconducting pairing (for details see [5]). By comparing the $T_{\mathrm{c}}(\delta)$ dependence for the Hubbard model: figure 5 (left panel) 
with $T_{\mathrm{c}}^{\max } \sim 280 \mathrm{~K}$, and for the $t$ - $J$ model: figure 7 (right panel) with $T_{\mathrm{c}}^{\max } \sim$ $180 \mathrm{~K}$, we observe a strong reduction of $T_{\mathrm{c}}^{\max }$ in the latter model due to taking into consideration a large contribution from the $\operatorname{Im} \Sigma_{\sigma}^{11}(\mathbf{k}, \omega)$ (see figure 6, right panel). At the same time, the large value of the $\delta_{\text {opt }} \simeq 0.33$ in the $t$ - $J$ model in comparison with the experimentally observed $\delta_{\text {opt }} \simeq 0.16$ and $\delta_{\text {opt }} \simeq 0.12$ in the Hubbard model shows that the $t-J$ model is incapable of properly reproducing the doping dependence of $T_{\mathrm{c}}$ since in the model the weight transfer between the Hubbard subbands under doping is neglected.

To conclude, the present investigations provide a microscopic theory for the superconducting pairing mediated by the AFM exchange interaction and spin-fluctuations induced by the kinematic interaction, characteristic of the Hubbard model. The singlet $d_{x^{2}-y^{2}}$-wave superconducting pairing was proved both for the original two-band $p-d$ Hubbard model and for the reduced effective one-band $t$ - $J$ model. These mechanisms of superconducting pairing are not observed in the fermionic models (for a discussion, see Anderson [19]) and turn out to be generic for cuprates. We believe that the proposed superconducting pairing is the relevant mechanism of high-temperature superconductivity in copper-oxide materials. 


\section{References}

1. Plakida N.M. High-Temperature Superconductivity. Springer-Verlag, Berlin, Heidelberg, 1995.

2. Anderson P.W., Science, 1987, 235, 1196; Anderson P.W. The Theory of Superconductivity in the High- $T_{\mathrm{c}}$ Cuprates. Princeton University Press, Princeton, 1997.

3. Plakida N.M., Yushankhai V.Yu., Stasyuk I.V., Physica C, 1989, 160, 80; Yushankhai V.Yu., Plakida N.M., Kalinay P., Physica C, 1991, 174, 401.

4. Izyumov Yu.A., Letfulov B.M., Intern. J. Modern Phys. B, 1992, 6, 321.

5. Plakida N.M., Oudovenko V.S., Phys. Rev. B, 1999, 59, 11949.

6. Plakida N.M., JETP Letters, 2001, 74, 36.

7. Emery V.J., Phys. Rev. Lett., 1987, 58, 2794; Varma C.M., Schmitt-Rink S., Abrahams E., Solid State Commun., 1987, 62, 681.

8. Plakida N.M., Hayn R., Richard J.-L., Phys. Rev. B, 1995, 51, 16599.

9. Feiner L.F., Jefferson J.H., Raimondi R., Phys. Rev. B, 1996, 53, 8751.

10. Yushankhai V.Yu., Oudovenko V.S., Hayn R., Phys. Rev. B, 1997, 55, 15562.

11. Plakida N.M., Anton L., Adam S., Adam Gh., ZhETF, 2003, 124, 367 (in Russian); [JETP, 97, 331].

12. Zubarev D.N., Usp. Fiz. Nauk, 1960, 71, 71 (in Russian); [Sov. Phys. Usp., 3, 320].

13. Beenen, J., Edwards D.M., Phys. Rev. B, 1995, 52, 13636.

14. Avella A., Mancini F., Villani D., Matsumoto H., Physica C, 1997, 282-287, 1757; Di Matteo T., Mancini F., Matsumoto H., Oudovenko V.S., Physica B, 1997, 230232, 915 .

15. Stanescu, T.D., Martin, I., Phillips Ph., Phys. Rev. B, 2000, 62, 4300.

16. Lokshin, K.A., Pavlov D.A., Putilin, S.N. et al., Phys. Rev. B, 2001, 63, 064511.

17. Harrison W.A. Electronic structure and the properties of solds. W.H.Freeman and Company, San Francisco, 1980.

18. Zhao G.-M., Singh K.K., Morris D.E., Phys. Rev. B, 1994, 50, 4112.

19. Anderson P.W., Adv. in Physics, 1997, 46, 3. 


\section{Теорія надпровідності в купратах}

\section{Н.М.Плакіда}

Об’єднаний інститут ядерних досліджень, 141980 Дубна, Росія

Отримано 11 липня, 2005

Розроблено мікроскопічну теорію надпровідного спарювання через антиферомагнітний (АФМ) обмін та спін-флуктуації в рамках ефективної $\boldsymbol{p}-\boldsymbol{d}$ моделі Хаббарда для площини $\mathrm{CuO}_{2}$. Доведено, що запізнюючі ефекти для АФМ обмінної взаємодії є неважливими і приводять до спарювання всіх електронів у зоні провідності та високої $\boldsymbol{T}_{\mathbf{c}}$, пропорційної до енергії Фермі. Спін-флуктуації, спричинені кінематичною взаємодією, дають додатковий внесок у спарювання $\boldsymbol{d}$-типу. Досліджується залежність $\boldsymbol{T}_{\mathrm{c}}$ від концентрації дірок та сталої гратки (чи тиску). Пояснено малий зсув $T_{\mathrm{c}}$ від ізотопів кисню. Отримані дані порівнюються з результатами для $\boldsymbol{t}-\boldsymbol{J}$ моделі.

Ключові слова: високотемпературна надпровідність, сильні електронні кореляції, модель Хаббарда, антиферомагнітна обмінна взаємодія, спін-флуктуації

PACS: 74.20.-z, 74.20.Mn, 74.72.-h 